\title{
Star Mass Inertia Dictates the Speed of Light
}

\author{
Weihong Qian ${ }^{1,2}$ \\ ${ }^{1}$ School of Physics, Peking University, Beijing, China \\ ${ }^{2}$ Guangzhou Institute of Tropical and Marine Meteorology, CMA, Guangzhou, China \\ Email: qianwh@pku.edu.cn
}

How to cite this paper: Qian, W.H. (2022) Star Mass Inertia Dictates the Speed of Light. Journal of High Energy Physics, Gravitation and Cosmology, 8, 184-194. https://doi.org/10.4236/jhepgc.2022.81014

Received: November 21, 2021

Accepted: January 15, 2022

Published: January 18, 2022

Copyright (C) 2022 by author(s) and Scientific Research Publishing Inc. This work is licensed under the Creative Commons Attribution International License (CC BY 4.0).

http://creativecommons.org/licenses/by/4.0/

\begin{abstract}
This paper indicated that Newton's law of gravitation is a statistical relation of two adjacent objects with a distance and Einstein's general relativity shows the internal connection of material distribution through space-time warping in our universe so general relativity is referred as a gravity theory. Here the paper gives an extension of general relativity in the sense of the metric theory of gravity which is consistent with Einstein's equivalence principle and generates a weak field approximation which extends Newtonian dynamics. Thus, the extended theory of gravity can infer that the light has a speed limit of photon escaping the mass inertia of a star.
\end{abstract}

\section{Keywords}

Newton, Einstein, Star Mass Inertia, Photon, Gravity, the Speed of Light

\section{Introduction}

Gravity is still incompletely understood and the most mysterious interaction among the fundamental forces of nature [1]. Newton and Einstein successively came up with the laws that express gravity for object motions in our universe. Newton's law of gravitation states that the attractive force is proportional to the mass product of two objects ( $m_{1}$ and $m_{2}$ ), varies as the inverse square of their distance $r$, and is multiplied by the gravitational constant $G$ [2].

$$
F=G \frac{m_{1} m_{2}}{r^{2}} .
$$

The Newtonian law can well illustrate that the motion of planets is regularly around the Sun, and the motion of moons is accurately around their planets in the Solar system. It also explains that the tidal phenomena are affected by the gravitational difference of the Moon on different parts of the Earth's oceans.

The law of gravitation originated from the empirical induction after analyzing 
a large number of astronomical observation data. It reflects a statistical relationship between "mass" and "force". This quantitative relation exists only in a finite interval distance between two adjacent objects because gravity does not exist at a considerable distance and a very short distance.

In Equation (1), the square of a distance is an area, so gravity is the mass product of two adjacent objects per unit area at a plane. The mass product of two adjacent objects over an area is the distribution of physical attributes, not stress or force [3]. Gravitation is not the transfer of momentum in direct contact between two objects, rather like an action at a distance. This action can only be explained by massless gravitons [4]. So far, however, gravitons have not been detected. Gravity cannot yet explain the opposite direction of rotation from neighboring planets in the Solar system.

Einstein (1911) studied the influence of gravitation on the propagation of light [5]. He (1916) then described that gravity is as a curved field of space-time [6]

$$
R_{\mu \nu}-\frac{1}{2} R g_{\mu \nu}=\frac{8 \pi G}{c^{4}} T_{\mu \nu} .
$$

In Equation (2): the term $R_{\mu v}$ is the Ricci curvature tensor, indicating how curved the space is; the term $g_{\mu v}$ is the $(3+1)$ four-dimensional space-time metric tensor; the term $T_{\mu \nu}$ is the mass-energy metric tensor for the distribution and motion of objects; the letter $R$ is the Ricci number of scalar curvature; the letter $G$ is the gravitational constant; and the letter $c$ is the speed of light in a vacuum. Einstein mapped the mass-energy distribution $\left(T_{\mu v}\right)$ of objects to the curvature of space-time $\left(R_{\mu v}, R\right)$ in the universe. Thus, Einstein argued that gravity is not a force at all. He described it as a space-time curvature associated with the distribution of mass and energy, no longer the direct interaction between two adjacent objects as Newton's law. Thus, Einstein's general relativity showed by the field Equation (2) is a gravity theory.

Two laws of Newton and Einstein described different processes. Newton first gives the relationship between the mass and force of two adjacent objects. Then the trajectory of an object's motion related to another is described through the "action force" but separated by a distance. Differently, the law of Einstein directly gives the equivalent relation between mass-energy distribution and the motion trajectory of objects in space. Therefore, the two laws described only the motion of objects in space rather than the nature of gravity. As summarized by Corda [7], even if Einstein's theory achieved a great success and overcame lots of experimental tests [8], it also showed some shortcomings and flaws which today advise theorists to ask if it is the definitive theory of gravity [9] [10]. Also, a long list of alternative theories to general relativity can be found in the review paper written by Clifton et al. [11].

\section{Nature of Gravity}

According to Equation (1), gravity formulated is a relation of action between two adjacent objects separated by a distance in the macroscopic world. How to explain the interaction between them? If there is only one object in the world, 
gravity does not exist. For two objects of the Earth $m_{1}$ and the Moon $m_{2}$ (or an apple over a tree), gravity exists between them separated by a vacuum distance. As an extreme, gravity will suddenly disappear from an infinity when the apple naturally falls to the Earth's surface. Gravity would not work if their distance is too far away or too close.

Gravity is a quantitative description of the moving mass inertia of all objects preserved since starting their astronomical evolution, rather than any interaction [3] [12]. Their mass inertias should be mostly equal if we pulverize all planets and natural satellites into as nebulas and scatter them on the current rotating plane of the Solar system. These nebulas can be seen as proto-Solar clouds at the moment when the Sun formed.

To illustrate the astronomical evolution of the Solar system, we first look at the spatial structure of the Milky Way galaxy. The Milky Way system is a spiral galaxy consisting of over 400 billion stars mainly concentrated along the four spiral high-density star bands. We infer that most stars along the four spiral star bands are moving toward the Galactic Center according to the similarity as the developing stage of a tropical cyclone over ocean [13]. At the last moment, the Galactic Center will become a "massive ball" in space because many star clusters have entered within the Galactic Center. The final disk structure of the Milky Way galaxy is similar to the Saturn and Saturn's rings. During the early stage, we similarly assume that there were four nebula spiral lines surrounding the Solar Core. After most nebulas entered the Solar Core along the four nebula spiral lines, remained nebulas formed four circular rings moving around the Sun when the Sun formed.

At that stage, 99\% masses of the Solar system had converged to the Sun and four encircled nebula rings with $98 \%$ angular momentum surrounded the Sun at the ecliptic plane. The rings had different nebula compositions depending on their distances to the Sun. Dense and rocky compositions were mostly concentrated near the Sun while most gaseous compositions were far away from the Sun. We assume that there were four nebula fluctuations surrounding the Sun so eight circular shear bands can be observed from " $\mathrm{A}$ " to " $\mathrm{H}$ " in Figure 1. Each

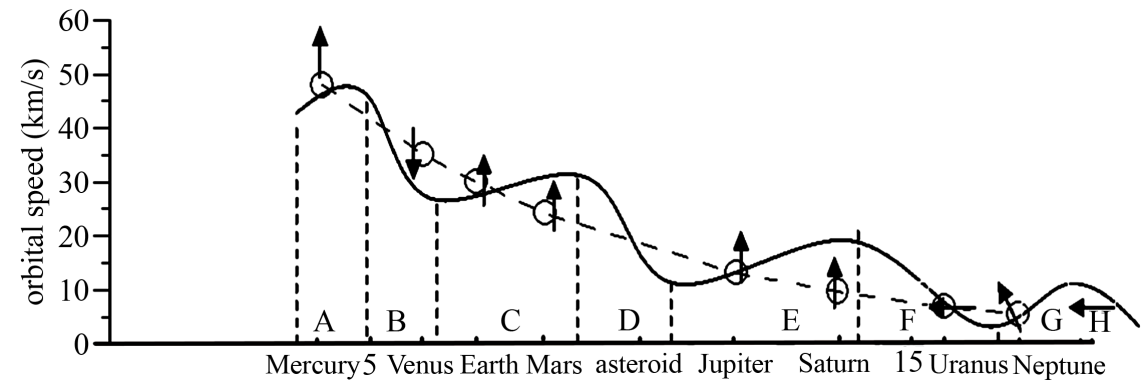

Figure 1. The orbital speed (long-dashed line, $\mathrm{km} / \mathrm{s})$ and distance $(D=3 \ln (10 R))$ of each planet (hollow circle) relative to the Sun $(D=0)$. The letter $R=1$ ( 1 astronomical unit, $15,000,000 \mathrm{~km}$ ) is the distance between the Sun and the Earth. Short-dashed lines separate different shear bands of nebula circular rings. Arrows denote different directions of planet rotation [3] [13]. 
circular band consisted of nebulas which can be seen as fluid-like motion (solid line in Figure 1). Therefore, small-scale vortices and their cores were generated with the same rotated direction at the same shear circular band. Two adjacent shear bands formed the opposite direction of nebula vortices and cores in their rotations. These cores were some embryos of planets. Within the same shear band, smaller embryos entered to larger ones or became as satellites of larger embryos. In the shear band "A," all embryos entered a large one so it became a planet named as the Mercury. In its outside band, the opposite shear band "B" formed the Venus with an opposite direction in rotation to the Mercury. The shear band " $\mathrm{C}$ " is relatively wider, so two planets (the Earth and the Mars), as well as several satellites (such as the Earth's moon) finally formed. The rotated direction of the Earth and the Mars as well as their satellites is opposite to that of the Venus but the same as the Mercury. The Asteroid belt with lots of embryos finally formed in the shear circular band "D". Similar to the shear band " $\mathrm{C}$ ", two giant planets of the Jupiter and the Saturn with their satellites generated in the shear band " $E$ " in the same direction of rotation. The Uranus and the Neptune formed in the same way in the shear bands "F" and "G". Beyond the shear band " $G$ ", there should have other shear bands such as " $\mathrm{H}$ " and vortices to form with different rotations. But their orbital periods of formed objects to the Sun are longer than those of the above planets. After planets and moons formed, their orbital speeds are gradually decreased with their distance to the Sun but their directions in rotation are opposite or different (Figure 1).

There should have superposed distributions of angular momentum at least two spatial levels. At a large level of the Solar system, angular momentums can explain the orbital motions of all objects surrounding the Sun. At a local level of the Earth-Moon system, their nebula angular momenta formed a planet and a satellite came from the shear band "C" near the left side in Figure 1. Gravity can be seen as the product of two linear mass densities $\left(F=G \frac{m_{1}}{r} \frac{m_{2}}{r}\right)$ during the forming stage of planet $\left(m_{1}\right)$ and moon $\left(m_{2}\right)$ along their distance $r$. The nebulas were locally circular moving around their center at a plane. Thus, there is no any gravity or attractive force on the plane, but only the inherent mass inertia of rotating objects nested by the rotated orbits of another larger spiral system or spatial level.

The action of gravity is a superficial phenomenon that looks likely a connection between two objects in space. Actually, the inherent mass inertia of an object has the property that resists change in its state of motion. The inherent mass inertia makes objects in a vacuum constant in shape and velocity. If we split an object into two parts for a distance, the inherent mass inertia must be changed. External forces are needed to change the inherent mass inertia of the object. In the universe, objects mostly move in circular orbits and are confined to a plane. Two forces can split the object into two parts, $m_{1}$ and $m_{2}$. To separate them, a mass centripetal force $\frac{m}{r} v^{2} n$ and a mass acceleration needed the force mak 
are written for each part.

$$
\begin{gathered}
\boldsymbol{F}_{1}=\frac{m_{1}}{r} v_{1}^{2} \boldsymbol{n}_{1}+m_{1} a_{1} \boldsymbol{k}_{1} \\
\boldsymbol{F}_{2}=\frac{m_{2}}{r} v_{2}^{2} \boldsymbol{n}_{2}+m_{2} a_{2} \boldsymbol{k}_{2}
\end{gathered}
$$

The cross product $\boldsymbol{F}_{1} \times \boldsymbol{F}_{2}$ of two forces acting on the object is a stress

$$
\boldsymbol{\tau}=\left(\frac{m_{1}}{r} v_{1}^{2} \boldsymbol{n}_{1}+m_{1} a_{1} \boldsymbol{k}_{1}\right) \times\left(\frac{m_{2}}{r} v_{2}^{2} \boldsymbol{n}_{2}+m_{2} a_{2} \boldsymbol{k}_{2}\right) .
$$

This stress is similarly like a description of mass-energy metric tensor and the evolutional tendency of object motions that can be derived after expanding into four terms from Equation (5). It is also very complex by using Equation (5) to describe the universal evolution acted by these forces. If there is no acceleration ( $a_{1}=a_{2}=0$, either expanding nor contracting of the universe) and the two vectors $\left(\boldsymbol{n}_{1}\right.$ and $\left.\boldsymbol{n}_{2}\right)$ are perpendicular, the result is

$$
\tau=4\left[\left(\frac{1}{2} m_{1} v_{1}^{2}\right) / r \cdot\left(\frac{1}{2} m_{2} v_{2}^{2}\right) / r\right] .
$$

The stress is a product of the kinetic energy linear density of two parts along the distance $r$. It can also write the similar form of Equation (1) as

$$
\tau=\frac{m_{1} m_{2}}{r^{2}}\left(v_{1} \cdot v_{2}\right)^{2} .
$$

Thus, gravity is a spatial mass-energy distribution in our universe. Here, one connection is clear from Newton's law locally or regionally for only two adjacent objects to Einstein's theoretical description globally for lots of objects. For the latter, a space-time curvature is caused by mass and energy. If comparing two Equations (1) and (7), the gravitational constant $G=\left(v_{1} \cdot v_{2}\right)^{2}$ is relied the speeds of two objects and only when the moving directions of two adjacent objects such as the Sun and the Earth are perpendicular. Equation (7) is a dynamical form of gravity while Equation (1) is a statistical form. It is clear that Equation (1) of the attractive force is only a special case from Equations (5) to (6). The above formulation showed that the vector field Equation (5) in this paper is an extension of the Einstein field Equation (2). However, the dynamic significance in Equation (5) is clearer than other theories of gravity.

On the other hand, Newton's traditional mechanics has widely and correctly used in our daily life. If the external force $\boldsymbol{m g}$ acts on a small ball $(m)$, it produces a momentum $(m v)$ change with time

$$
\frac{\mathrm{d}(m \boldsymbol{v})}{\mathrm{d} t}=m \boldsymbol{g} \text {. }
$$

We choose the Earth as an inertial frame of reference. The small ball (or an apple) will have acceleration $\boldsymbol{g}$. It makes the apple's speed $\boldsymbol{v}(t)$ increasing with time $t$. The vector denotes the direction from the apple to the Earth. The tree branch has a force $-\mathbf{m g}$ to the apple keeping in the air. 
When the apple hangs on a tree branch, its potential energy related to the Earth's inertial acceleration $\boldsymbol{g}$ is stored. How is this potential energy stored on the apple? The growth of apple is an accumulation of water molecules and other nutrient molecules transferred from the root along the trunk and branch of the tree to growing fruit. A force $f_{i}$ exerts on a molecule (mass $\varepsilon_{i}$ ) that is originated from the Earth during the growing season.

$$
\boldsymbol{f}_{i}=\varepsilon_{i} \boldsymbol{g} .
$$

The microscopic dynamics of apple tree growth is photosynthesis indicated by the force $f_{i}$. When an apple hangs over a branch at the height $h_{0}$, it accumulates potential energy $\varepsilon_{i} g h_{0}$ related to the ground. Thus, an apple falling to the ground results from the natural return of all the molecules. This natural return to the Earth is due to the inherent mass inertia of the apple, which can be expressed by gravity. The ripening apple on a branch stored not only the potential energy, but also the biological energy structured by all of apple moleculars.

Newton's law of universal gravitation describes only one segment of the life cycle of an apple tree from the ripening apple on a tree branch to fall the Earth naturally. If we plant an apple tree on the surface of the Earth in the same soil environment, but without giving sunlight, the apple cannot grow on the tree. Therefore, seasonal changes in photosynthesis as forcing are essential to apple tree growth. The life cycle of an apple tree can be described as that: 1) water and nutrients rise from the root along the trunk and branches to blossoms during spring under sunlight; 2) in summer months, the apple grows up under the photosynthesis; 3 ) the ripening apple in autumn falls naturally with the inertial acceleration $\boldsymbol{g}=9.8 \mathrm{~m} / \mathrm{s}^{2}$ from the branches to the ground at the moment due to the inherent inertia of all molecules of the apple; and 4) the apple on the ground will be rotted in winter, backing water and nutrients into the Earth's soil. Year after year, apples in the natural environment mature and fall to the ground from their branches. Sunlight is the fundamental driver for storing the potential and biological energies of apple during this cycle.

\section{Nature of Light}

Different particle speeds exist in the natural flows of water, air, and light. The speed of light is the fastest. The relation between the mass change $\Delta m$ and the energy change $\Delta E_{c}$, which described by Einstein [14], is like as

$$
\Delta E_{c}=\Delta m c^{2} .
$$

It expresses the relationship of equivalent measure between mass and energy as objects accelerate to the speed of light.

In the real world, the kinetic energy $E$ of a body has a relation with its mass $m$ and velocity $|v|$,

$$
E=\frac{1}{2} m|v|^{2}
$$

When the velocity of an object gradually goes to the speed of light, we consid- 
er the inherent relationship between the change in mass $\Delta m$ and the change in energy $\Delta E$ as

$$
\Delta E=\frac{1}{2} \Delta m|v|^{2}
$$

If we take a mass $\Delta m$ from an object and accelerate it to the speed of light, the energy $\Delta E$ could be received. Once the mass becomes photons, they should have momentum and energy. Photovoltaics rely on the momentum and energy of photons. Whether a photon has mass is a long-standing issue. Newton's particle theory of light does not say for sure whether a photon has a rest mass, while Einstein categorically denied that photons had any rest mass. The reason for this is changing scales in a weak field approximation of general relativity.

Under this approximation but related to the speed of light $c$ as a constant, Einstein derived the space-time transition relationship between the two inertial systems. On a direction $x$ of space, the Lorentz transformation with time $t$ is like [15],

$$
x^{\prime}=\gamma(x-v t)
$$

by changing scales

$$
\gamma=\frac{1}{\sqrt{1-v^{2} / c^{2}}}
$$

The scale $\gamma=1$, only when the velocity $v=0$, so it degrades as the Galilei transformation. Thus, it is a parameter (an elastic ruler) varied from the speed of an object relative to the speed of light. But Einstein introduced it into the relativistic momentum,

$$
\boldsymbol{p}(v)=m(v) \boldsymbol{v}=\gamma m_{0} \boldsymbol{v},
$$

where, the letter $m_{0}$ is the rest mass of an object. In the low velocity of object, $\gamma=\frac{1}{\sqrt{1-v^{2} / c^{2}}} \rightarrow 1$, Newton's second law of motion $\boldsymbol{f}=m_{0} \frac{\mathrm{d} \boldsymbol{v}}{\mathrm{d} t}$ is approximately satisfied. The moving mass $m(v)$ is directly associated with the rest mass $m_{0}$ and the velocity $v$ as the following relation

$$
m(v)=\frac{m_{0}}{\sqrt{1-v^{2} / c^{2}}}
$$

Newton's second law is an approximation to the theory of relativity because the speed of light $c$ is a given constant and ordinary objects cannot travel to the speed of light. As an object approaching the speed of light, its denominator tends to be infinitesimal. It implies that the mass of a photon approaching the speed of light tends to infinity. If so, each Sun's photon that reaches the Earth has a tremendous amount of momentum or moving mass $m(v)$. Thus, the sunlit side of the Earth has long been battered beyond recognition by the Sun's photons. However, this situation has not been observed. Furthermore, accelerating photons cannot travel faster than the constant speed of light in the Solar system. 
Otherwise, this mathematical expression in Equation (16) does not make any physical sense. So, the photon's rest mass must be zero $\left(m_{0}=0\right)$. This is a mathematical singularity that has no physical meaning in the real world. Inversely, Equation (16) can write as

$$
m_{0}=m(v) \sqrt{1-v^{2} / c^{2}} .
$$

The initial mass $m_{0}$ of an object is minimal, even reaching zero but is not equal to zero when it approximates the speed of light $m(v \rightarrow c)$.

The experimental results showed that photons have mass. In the last three decades, detected rest mass of photons was varied from $2 \times 10^{-47} \mathrm{~g}$ to $1.2 \times 10^{-51}$ $\mathrm{g}$ [16] and $1.5 \times 10^{-52} \mathrm{~g}$ [17]. The masses of the oxygen and iron atoms are 2.657 $\times 10^{-23} \mathrm{~g}$ and $9.288 \times 10^{-23} \mathrm{~g}$, respectively. The static masses of electron and neutron are $9.109 \times 10^{-28} \mathrm{~g}$ and $1.6749 \times 10^{-24} \mathrm{~g}$, respectively. So, a photon is the lightest particle with its mass approaching zero but not equal to zero. It shows that the photon when it accelerates to the speed of light requires a minimal mass.

Returning to Equation (12), the mass of an object as it accelerates towards the speed of light is examined

$$
\Delta m=2 \frac{\Delta E}{|v|^{2}} .
$$

If the velocity of an object increases to the speed of light, its energy is inevitable so its mass is not zero. What emerges is a photon with a high speed and an infinitely minimal mass. The faster a photon goes, so the less mass is.

Here we examine an apple with its mass $m$ on a branch at the height $h$ to see how it can become a photon. The external force $m a_{h}$ acts on the apple with acceleration $\boldsymbol{a}_{h}$. The first goal is to overcome the inertial acceleration $\boldsymbol{g}$ exerted on the apple. Actually, the goal is preventing all molecules of the apple to return the Earth. When

$$
\boldsymbol{a}_{h}-\boldsymbol{g}=\frac{\mathrm{d} \boldsymbol{v}}{\mathrm{d} t}=0,
$$

the apple leaves the branch with a rest state relative to the Earth without freely falling to the ground. And then, we put more force on the apple to accelerate it,

$$
\boldsymbol{a}_{m}>\boldsymbol{a}_{h}=\boldsymbol{g} .
$$

The second goal is accelerating the apple to escape the Earth's inertial acceleration $\boldsymbol{g}$ within a time interval $T$. Finally, the speed of the apple reaches the velocity maximum $\left(V_{M}\right)$

$$
\boldsymbol{v}=\int_{0}^{T} \boldsymbol{a}_{m} \mathrm{~d} t \rightarrow V_{M} .
$$

It becomes a photon with its mass approaching zero. One can build a device, such as a nuclear reactor, to make its mass approaching zero with the speed of light.

The Sun is a natural nuclear reactor and has large mass, so the acceleration $\boldsymbol{g}_{\Theta}$ on the surface of the Sun is tremendous. Nuclear reactions produce lots of 
photon due to being relative motions between the different layers inside the Sun. The photon at the speed of light carries substantial energy. The ability of producing photons is proportional mass $m_{\Theta}$ of a star and relative motions between inner layers. According to radiometric dating and other sources of evidence, the Earth formed about 4.54 billion years ago but after the Sun formed [13]. If the initial mass $M_{\Theta}$ of the Sun was double as the present $\left(M_{\Theta}=2 m_{\Theta}\right)$, the escape velocity of photons from the original Sun surface should be double faster than the current speed of light. The dimming of a star brightness shows a declining ability to excite photons due to weakening relative motion and decreasing mass. Thus, the star degenerates into a black hole which shows that produced photons cannot escape the inherent inertial acceleration of the star. In the universe, the escape velocity of photons is varying as it leaves the surfaces of different mass stars. Speeds of light in the vacuum space are not a constant because there are many other mass stars. This result is different from the special relativity implying that the speed of any moving object cannot exceed that of light in a vacuum [18] but as speculated by Magueijo [19].

\section{Conclusions and Discussion}

From macro to micro aspects, this paper reasoned if the existence of gravity is based on a novel approach of mathematical physics. The results showed that gravity is a regional mass-energy distribution or a local action of two adjacent objects to overcome their inherent mass inertias as statistically formulated by Newton's law. Globally, gravity presents the space-time curvature characteristics nested by multi-level rotating structures as described by Einstein's field equation. Therefore, the inherent mass inertia and angular momentum are two physical quantities in the universal evolution. Changing in the current mass inertia and angular momentum requires external force. This study was an extension of general relativity in the sense of the metric theory of gravity which is consistent with Einstein's equivalence principle and generates a weak field approximation which extends Newtonian dynamics. Among all microscopic particles, photons have minimal mass. In the universe, stars are the source exciting photons, because there are relative motions between inner layers in the glowing star. The speed of light excited at the surface of a star is directly proportional to the star's mass and the relative motion intensity inside the star. Therefore, stars with different masses and aging excite different speeds of light.

The driving forces of tidal phenomena are not the difference of gravity from the Moon to different surfaces of the Earth, rather than the inherent mass inertia of marine fluid. Like the Moon, every water molecule in the ocean has its inherent mass inertia relative to the Earth. Taking the Earth as the inertial frame of reference, the center of the Earth is at an elliptical focus of the Moon's orbit. Also, the center of the Earth is at an elliptical focus of the molecule climate motion of oceanic water. The difference is that the ocean is a thin fluid layer outside the Earth. There is direct contact between oceanic water molecules affected by mul- 
ti-scale thermo-dynamical disturbances. The planetary-scale general form of seawater can be regarded as a "sponge" that wraps the solid Earth. The sponge has the inherent mass inertia since beginning the evolution of the Earth-Moon system. Therefore, the ocean is characterized by normal tides as the Moon moving around the Earth.

The atmosphere is another layer of sponge covering the solid Earth, which has much less dense than the ocean. The relationship between the atmospheric sponge and the central Earth is like Saturn's rings and the central Saturn. The atmospheric tides have been calculated on average by observing atmospheric variables for many years [13]. It has a temporal climatology with daily cycle changing hourly and annual cycle changing seasonally. It is also called the reference atmosphere with high spatial and temporal resolutions. Global atmospheric variables observed every day and every hour could be decomposed into two parts of reference atmosphere and relative disturbance. The reference atmosphere is the climate tides or the temporal climatology [13]. The relative disturbance is a crucial factor in producing all kinds of extreme weather [20].

\section{Acknowledgements}

The author wishes to thank the editor and anonymous reviewers whose constructive suggestions greatly improved the manuscript. This work is supported by the innovative R\&D project in Guangzhou Province in China (2019ZT08G669) and the National Natural Science Foundation of China (Grant Number: 41775067).

\section{Conflicts of Interest}

The author declares no conflicts of interest regarding the publication of this paper.

\section{References}

[1] Butto, N. (2020) New Theory to Understand the Mechanism of Gravitation. Journal of High Energy Physics, Gravitation and Cosmology, 6, 462-472. https://doi.org/10.4236/jhepgc.2020.63036

[2] Newton, I. (2020) Mathematical Principles of Natural Philosophy. Filozofski Vestnik, 41, 9-79.

[3] Qian, W.H. (2011) Who Is Driving Climate Change? Science Press, Beijing.

[4] Misner, C.W., Thorne, K.S. and Wheeler, J.A. (1973) Gravitation. W. H. Freeman and Company Limited, Reading.

[5] Einstein, A.A. (1911) On the Influence of Gravitation on the Propagation of Light. Annalen der Physik, 35, 898-901.

[6] Einstein, A.A. (1916) The Foundation of the General Theory of Relativity. Annalen der Physik, 49, 769-822. https://doi.org/10.1002/andp.19163540702

[7] Corda, C. (2009) Interferometric Detection of Gravitational Waves: The Definitive Test for General Relativity. International Journal of Modern Physics D, 18, 22752282. https://doi.org/10.1142/S0218271809015904

[8] Misner, C.W., Thorne, K.S. and Wheeler, J.A. (1973) Gravitation (Physics Series). 
W.H. Feeman and Co., New York.

[9] Capozziello, S. and Francaviglia, M. (2008) Extended Theories of Gravity and Their Cosmological and Astrophysical Applications. General Relativity and Gravitation, 40, 357-420. https://doi.org/10.1007/s10714-007-0551-y

[10] Sotiriou, T.P. and Faraoni, V. (2010) $f(R)$ Theories of Gravity. Reviews of Modern Physics, 82, 451-497. https://doi.org/10.1103/RevModPhys.82.451

[11] Clifton, T., Ferreira, P.G., Padilla, A. and Skordis, C. (2012) Modified Gravity and Cosmology. Physics Reports, 513, 1-189. https://doi.org/10.1016/j.physrep.2012.01.001

[12] Qian, W.H. (2020) The Real World: Structure and Anomaly in Universal System. Jiangsu Phoenix Science and Technology Press, Nanjing.

[13] Qian, W.H. (2017) Temporal Climatology and Anomalous Weather Analysis. Springer, Singapore.

[14] Einstein, A.A. (1923) Does the Inertia of a Body Depend upon Its Energy Content? The Principle of Relativity. Translation by George Barker Jeffery and Wilfrid Perrett, Methuen and Company, Ltd., London, 35-65.

[15] Einstein, A.A. (1920) Relativity: The Special and General Theory. Translated by Lawson, R.W., Henry Holt and Company, New York.

[16] Luo, J., Tu, L.C., Hu, Z.K. and Luan, E.J. (2003) New Experimental Limit on the Photon Rest Mass with a Rotating Torsion Balance. Physical Review Letters, 90, Article ID: 081801. https://doi.org/10.1103/PhysRevLett.90.081801

[17] Tu, L.C. and Luo, J. (2006) Progress in Experimental Tests of the Photon Rest Mass. Physics, 35, 779-784.

[18] Wang, L.J., Kuzmich, A. and Dogariu, A. (2000) Gain-Assisted Superluminal Light Propagation. Nature, 406, 277-279. https://doi.org/10.1038/35018520

[19] Ellis, G. (2003) Einstein Not yet Displaced. Nature, 422, 563-564. https://doi.org/10.1038/422563a

[20] Qian, W.H., Du, J. and Ai, Y. (2021) A Review: Anomaly-Based versus Full-FieldBased Weather Analysis and Forecasting. Bulletin of the American Meteorological Society, 102, E849-E870. https://doi.org/10.1175/BAMS-D-19-0297.1 